\title{
Hormigón ligero armado - Fabricación,
propiedades, ejecución, resistencia y densidad aparente del hormigón ligero armado
}

H. WEIGLER Y S. KARL, Darmstadt

Betonstein-Zeitung, n. ${ }^{\circ} 10$, octubre 1970 , págs. $604-609$

\begin{abstract}
El hormigón ligero armado registra un desarrollo muy pronunciado no sólo en la República Federal Alemana, sino también en el mundo entero. Prácticamente no existe un sector de la construcción en el que no se utilicen sus ventajas. Debido a las propiedades favorables del material, el hormigón ligero armado abre nuevas posibilidades al conjunto de la construcción en hormigón. Hoy en día, es posible, con las instalaciones normales existentes, tanto en las fábricas de hormigón como en la obra, preparar económicamente hormigones ligeros de las calidades LB 120 a LB 450 con densidades aparentes en el hormigón de 1,2 a $1,8 \mathrm{t} / \mathrm{m}^{3}$. La condición más importante para la elaboración de hormigón ligero es, en primer lugar, el conocimiento exacto de los valores característicos del material; también conviene conocer la peculiaridad y naturaleza de todos los componentes del hormigón ligero, y su combinación con el acero bajo la forma de material de construcción mixto.

Para proporcionar al mundo técnico una representación resumida del estado de las investigaciones y de la aplicación práctica del hormigón ligero armado, en la primavera de 1971 aparecerá en el Bauverlag, Wiesbaden, bajo el titulo "Hormigón ligero armado fabricación-propiedades-ejecución", la publicación de una obra. Como autor figura el profesor Dr. Ing. H. Weigler y como colaborador el Dipl. Ing. Karl, de la Escuela Técnica Superior de Darmstadt. El profesor Weigler se ocupa desde haca años de esta especialidad y es conocido en el mundo entero por sus diversos trabajos de investigación y publicaciones.
\end{abstract}

A fin de procurar una impresión de esta obra, se da a continuación un resumen del contenido y el apartado "Resistencia y densidad aparente del hormigón ligero armado".

\section{RESISTENCIA Y DENSIDAD APARENTE DEL HORMIGON LIGERO ARMADO}

\section{Resistencia a la compresión y densidad aparente}

La resistencia a la compresión y la densidad aparente son las dos magnitudes características más importantes de una mezcla de hormigón ligero y, por lo tanto, antes de empezar la obra se comprobarán siempre realizando los oportunos ensayos de calidad. 
Como quiera que los hormigones ligeros aquí tratados se componen de $\approx$ un $70 \%$ de volumen de áridos, su densidad aparente dependerá, en primer lugar, de la densidad aparente de los gránulos de árido en su conjunto. Además de la clase de áridos, en contraposición a áridos normales, en este aspecto desempeña también un papel importante la composición granulométrica, ya que las densidades aparentes de los gránulos de las diversas fracciones pueden diferenciarse notablemente. La mayoría de las veces, al crecer el diámetro del gránulo disminuye la densidad aparente. Una granulometría más rica en finos o un diámetro menor en el tamaño máximo conducen, en general, a una mayor densidad aparente del hormigón y también, la mayoría de las veces, a una mayor resistencia. Una sustitución de finos de los áridos ligeros por arena natural más pesada aumenta la densidad aparente del hormigón. Un hormigón ligero que, por ejemplo, contiene $20 \%$ en volumen de árido con arena natural en vez de arena de árido ligero, es, $\approx 0,2$ $\mathrm{kg} / \mathrm{dm}^{3}$ más pesado.

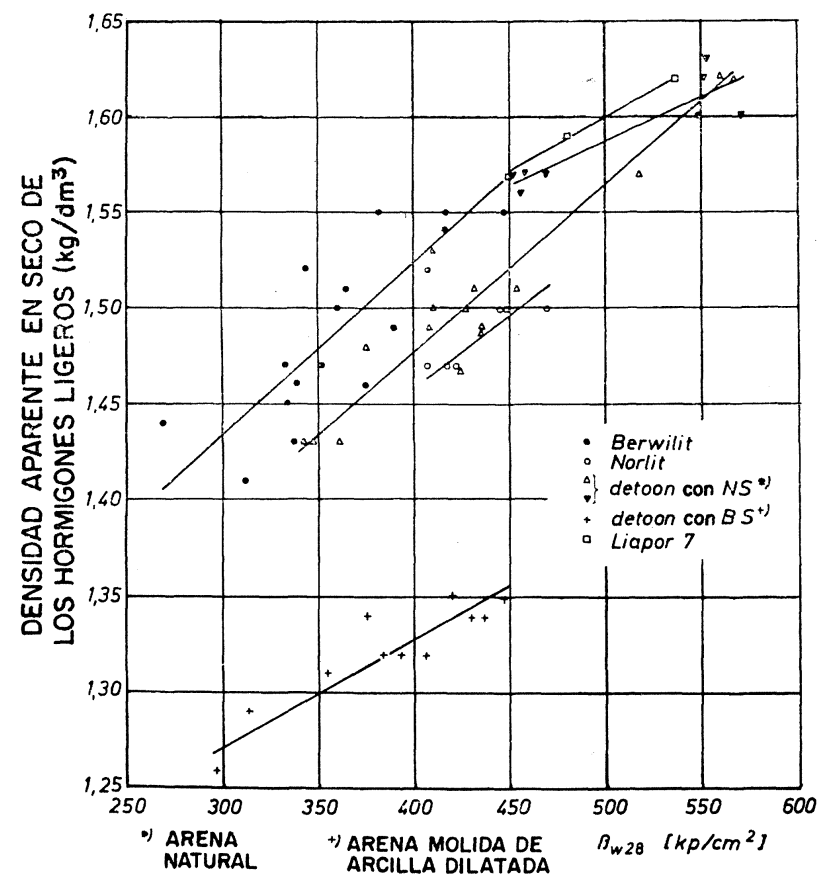

Fig. 1.-Relación entre la resistencia a la compresión del hormigón ligero y la densidad aparente en seco de hormigones ligeros preparados con áridos alemanes.

Como quiera que una adición de arena natural repercute favorablemente en la trabajabilidad del hormigón fresco, a igualdad de consistencia puede ser menor la demanda de agua. Esto conduce a un aumento condicionado de la resistencia. Con igual relación "activa" agua-cemento o bien igual relación huecos-cemento, la resistencia queda, sin embargo, prácticamente inalterada. (Como "huecos" se consideran las partes del hormigón no ocupadas por los gránulos del árido y el cemento. Se compone del agua que existe en la pasta de cemento, de los poros producidos en la compactación y de las oclusiones de aire introducidas artificialmente.) 
La figura 1 muestra la relación entre la resistencia a la compresión $\beta_{w 28}$, y la densidad aparente en seco de hormigones ligeros preparados con áridos que en la República Federal de Alemania se fabrican en producción continua y son adecuados para hormigones con resistencias superiores a los $300 \mathrm{kp} / \mathrm{cm}^{2}$. Las mezclas contienen generalmente arena natural en una proporción del $20 \%$ en volumen de los áridos. Los hormigones fabricados con arcilla dilatada "detoon" sin adición de arena natural, en los que la granulometría $<2 \mathrm{~mm}$ se compone principalmente de arena procedente de arcilla dilatada molida, llaman la atención por su reducida densidad aparente en relación con su resistencia.

A resistencias crecientes; en todos los áridos examinados aumenta la densidad aparente del hormigón en una medida aproximadamente igual. Esto se basa en que al aumentar la resistencia disminuye el contenido en árido ligero en favor de la parte pesada de pasta de cemento.

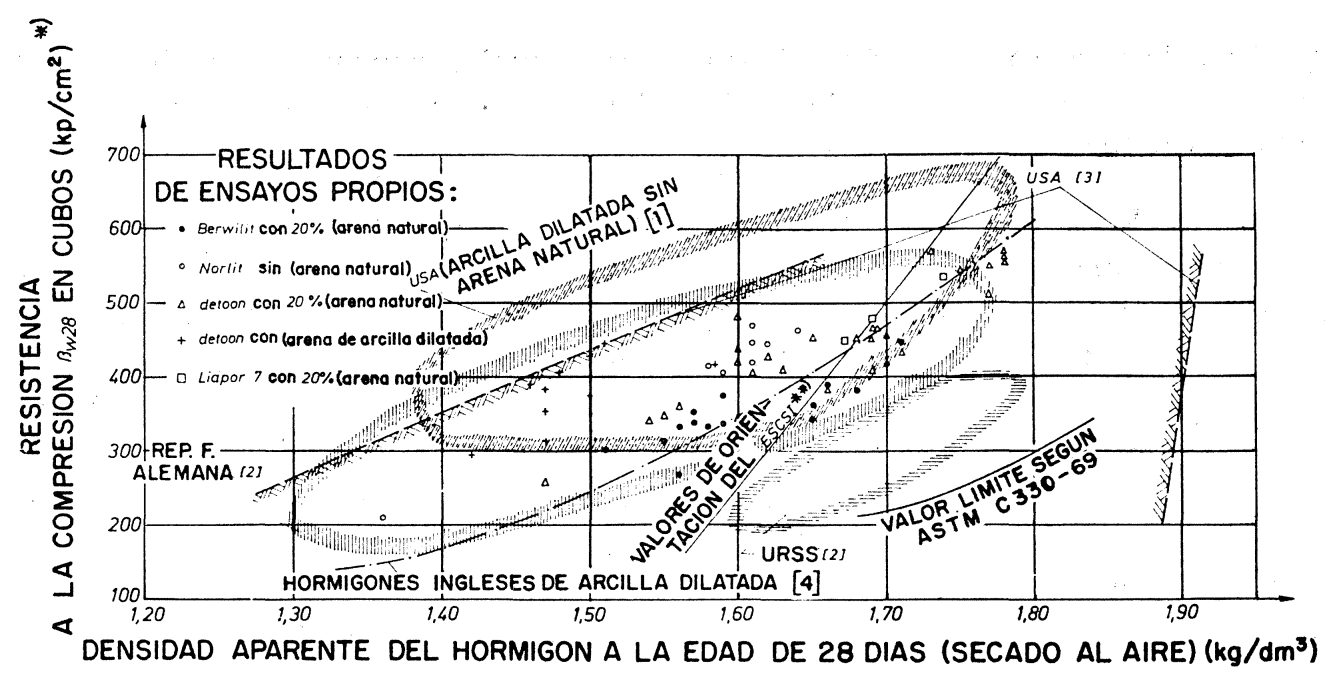

Fig. 2.-Comparación de las resistencias y las densidades aparentes de hormigones ligeros alemanes y de otros países.

*) Los valores americános de resistencia obtenidos según el método ASTM se aumentan en la adaptación a las relaciones alemanas en $\approx 20 \%$.

**) Esquisto expandido, Instituto de Arcilla y Esquistos, Washington D.C.

En la figura 2 se comparan las resistencias a la compresión alcanzadas a los 28 días con distintas densidades aparentes de hormigones ligeros del país y del extranjero. En la República Federal de Alemania, la resistencia máxima conseguida es de $\approx 550 \mathrm{kp} / \mathrm{cm}^{2}$ con densidades aparentes comprendidas entre 1,7 y $1,8 \mathrm{~kg} / \mathrm{dm}^{3}$. Esta resistencia a la compresión puede alcanzarse con algunos áridos ligeros americanos con densidades aparentes de 1,5 a $1,6 \mathrm{~kg} / \mathrm{dm}^{3}$. Las calidades máximas de hormigones conseguidas en USA con áridos de arcilla dilatada manifiestan resistencias de casi $700 \mathrm{kp} / \mathrm{cm}^{2}$ con densidades aparentes comprendidas entre 1,7 y $1,8 \mathrm{~kg} / \mathrm{dm}^{3}$. Frente a esto, los hormigones ligeros fabricados en la URSS parecen ser más desfavorables, en conjunto. La resistencia a la compresión no pasa de $400 \mathrm{kp} / \mathrm{cm}^{2}$. Las densidades aparentes correspondientes son, con 1,7 a $1,8 \mathrm{~kg} / \mathrm{dm}^{3}$, relativamente elevadas. 
Con los áridos y cementos de que se dispone en los distintos países se fabrican o pueden fabricarse hormigones ligeros de las siguientes calidades y densidades:

\begin{tabular}{|l|c|c|c|}
$\begin{array}{c}\text { Calidad del } \\
\text { hormigón }\end{array}$ & \multicolumn{3}{|c|}{ DEnSIDADES APARENTES } \\
& $\begin{array}{c}\text { Rep. Fed. de } \\
\text { Alemania }\end{array}$ & USA & URSS \\
LB 225 & $1,3-1,5$ & 1,3 & $1,6-1,7$ \\
LB 300 & $1,4-1,7$ & $1,3-1,6$ & $1,7-1,8$ \\
LB 450 & $1,5-1,8$ & $1,4-1,7$ & - \\
LB 600 & - & $1,7-1,8$ & -
\end{tabular}

La economía de peso con respecto a hormigones normales comparables es del 25 al $40 \%$ en hormigones ligeros con áridos de arcilla dilatada y esquisto dilatado, con y sin adición de arena natural.

La clase de árido ligero ejerce sobre el desarrollo de la resistencia y endurecimiento posterior una influencia que no puede señalarse a priori cuantitativamente. Por consiguiente, tampoco es posible señalar un índice de validez general para la relación resistencia inicial - resistencia a los 28 días: El desarrollo de la resistencia de hormigón ligero conservado constantemente en húmedo corresponde entonces sólo a la del hormigón normal si la resistencia final que se pretende se halla en una zona en la que la resistencia y el comportamiento a la deformación de los áridos aún no son decisivos para que falle el hormigón (caso a). En el otro caso (b), la resistencia del hormigón crece más lentamente que la resistencia de la pasta de cemento. En estas circunstancias, a los 7 días puede haberse conseguido prácticamente ya la resistencia definitiva.

La tabla 1 presenta, para la comparación, las resistencias a la compresión de mezclas de hormigón ligero con diferentes áridos y de mortero normalizado (RILEM-CEM) a la edad de 7 y 28 días; todas las mezclas se hicieron con el mismo cemento. En los hormigones ligeros se trata de "mezclas standard" según el apartado 7.3.6 de las "Directrices provisionales". Con un contenido de cemento de $\approx 350 \mathrm{~kg} / \mathrm{m}^{3}$, la relación huecos-cemento fue de $\approx 0,50$. Como la calidad de las pastas de cemento es en todos los casos prácticamente igual, por tener una relación agua-cemento constante, las resistencias de los hormigones de las diversas mezclas tienen que ser una consecuencia de las distintas propiedades de los gránulos de los áridos ligeros. La parte de carga transmitida por los áridos aumenta, por consiguiente, en el orden indicado en la tabla 1, de arriba hacia abajo. Esta tendencia está en consonancia con la creciente densidad aparente de los gránulos gruesos. Sólo los tres hormigones ligeros con áridos "altamente resistentes" manifiestan del $7 .^{\circ}$ al 28. ${ }^{\circ}$ día un aumento de resistencia similar al correspondiente mortero normalizado u hormigón normal. En los demás, la docilidad de los gránulos del árido disminuye claramente el aumento de resistencia. También aquí se manifiesta que el grado de destrucción por compresión no representa una magnitud característica del comportamiento resistente del árido en el hormigón. 
T A B L A 1

Relación entre la resistencia a la compresión a la edad de 7 días y la resistencia a la compresión a los 28 días, en "mezcla standard" con distintos áridos ligeros y mortero normalizado con el mismo cemento.

\begin{tabular}{|c|c|c|c|c|}
\hline $\begin{array}{l}\text { Clase de } \\
\text { árido }\end{array}$ & $\begin{array}{l}\text { Grado de destrucción } \\
\text { por compresión }\end{array}$ & $\begin{array}{l}\text { Densidad aparente de } \\
\text { los gránulos gruesos } \\
(\sim 7 / 15 \mathrm{~mm})\end{array}$ & $\begin{array}{l}\text { Relación hue- } \\
\text { cos/cemento }\end{array}$ & $\beta_{w 1} / \beta_{w 28}$ \\
\hline $\begin{array}{l}\text { Arcilla expandida } \\
\text { Liapor } 4\end{array}$ & 0,56 & 0,8 & 0,51 & $178 / 198=0,90$ \\
\hline $\begin{array}{l}\text { Arcilla expandida } \\
\quad \text { Liapor } 5\end{array}$ & 0,21 & 1,0 & 0,50 & $238 / 255=0,94$ \\
\hline $\begin{array}{l}\text { Esquisto expandido } \\
\text { Berwilit }\end{array}$ & 0,51 & 1,0 & 0,50 & $290 / 315=0,92$ \\
\hline $\begin{array}{l}\text { Esquisto expandido } \\
\text { Norlit }\end{array}$ & 0,31 & 1,2 & 0,52 & $302 / 363=0,83$ \\
\hline $\begin{array}{l}\text { Arcilla expandida } \\
\text { detoon }\end{array}$ & 0,42 & 1,4 & 0,49 & $359 / 423=0.85$ \\
\hline $\begin{array}{l}\text { Arcilla expandida } \\
\text { Liapor } 7\end{array}$ & 0,18 & 1,4 & 0,47 & $376 / 472=0,80$ \\
\hline $\begin{array}{cc}\text { Mortero } & \text { normalizado } \\
\text { según } & \text { RILEM-CEMM }\end{array}$ & - & - & 0,50 & $\begin{array}{c}\mathrm{N}_{7} / \mathrm{N}_{28} \\
394 / 496=0,79\end{array}$ \\
\hline
\end{tabular}

El endurecimiento ulterior (relación $\beta_{\infty} / \beta_{28}$ ) depende de la clase de cemento empleada. Unicamente es igual al correspondiente aumento en hormigón normal cuando la resistencia definitiva se halla por debajo del límite en el que aparecen los gránulos del árido como componente más débil del material compuesto hormigón (caso a).

En el caso b, la medida del endurecimiento ulterior depende, además de las propiedades del cemento y de los áridos, del contenido de cemento. Con aumento creciente de cemento corresponden a iguales resistencias iniciales resistencias definitivas menores. En Hormigón Berwilit, por ejemplo, la resistencia entre 7 y 28 días, con un contenido de cemento de $335 \mathrm{~kg} / \mathrm{m}^{3}$, aumentó $\approx$ un $20 \%$, mientras que con un contenido de cemento de $355 \mathrm{~kg}$ sólo aumentó un $11 \%$. Con contenidos de cemento aún mayores se refuerza esta tendencia, como ya se ha explicado, debido a la creciente influencia de la resistencia propia y de la rigidez del gránulo.

\section{Resistencia a la tracción por flexión y a la tracción por hendimiento}

La resistencia del hormigón con un esfuerzo de tracción se determina por la resistencia a la tracción de los áridos, la resistencia a la tracción de la pasta de cemento o mortero aglutinantes y la calidad de la unión entre los gránulos de los áridos y la pasta de cemento. A igualdad de resistencia a la compresión del hormigón, la pasta de cemento en el hormigón ligero presenta generalmente una resistencia a la compresión mayor que en el hormigón normal. El comportamiento de la unión íntima entre gránulos del árido y pasta de cemento es, la mayoría de las veces, especialmente favorable en el hormigón ligero debido a la superficie rugosa y, en parte, porosa de los áridos. Las acumulaciones de agua en la superficie de los gránulos, que con áridos normales compactos 
perjudican la adherencia, no tienen lugar en este caso, ya que el agua sobrante la absorben los propios gránulos. Por estas causas, los hormigones ligeros, en la zona inferior de resistencia, pueden desarrollar resistencias a la tracción especialmente elevadas en relación a la resistencia a la compresión. Como muestra la figura 3, incluso con áridos de arcilla dilatada, de alta resistencia, redondeados y relativamente lisos, ya a partir de una resistencia a la compresión de menos de $400 \mathrm{kp} / \mathrm{cm}^{2}$ los gránulos están tan fuertemente aglutinados en el esqueleto del mortero, que en el ensayo de tracción por flexión no se desprenden, sino que se rompen. Su resistencia propia es pequeña frente a las fuerzas desarrolladas por la adherencia. Con un aumento en la calidad del hormigón no crece la fuerza de tracción transmisible por los áridos. La fuerza de tracción admisible en conjunto sólo puede aumentarse por una creciente resistencia del mortero. Frente a esto, la resistencia propia de los gránulos es notablemente mayor en la mayoría de los áridos normales. En este caso, sólo con una calidad de hormigón de unos $600 \mathrm{kp} / \mathrm{cm}^{2}$ se rompen todos los gránulos. En la zona de resistencias situada por debajo de esta cifra los gránulos del árido se atraen en medida creciente al mejorar las propiedades de la unión íntima; por lo que aquí la resistencia a la tracción ha de aumentar más rápidamente que en el hormigón ligero, al crecer la resistencia a la compresión.

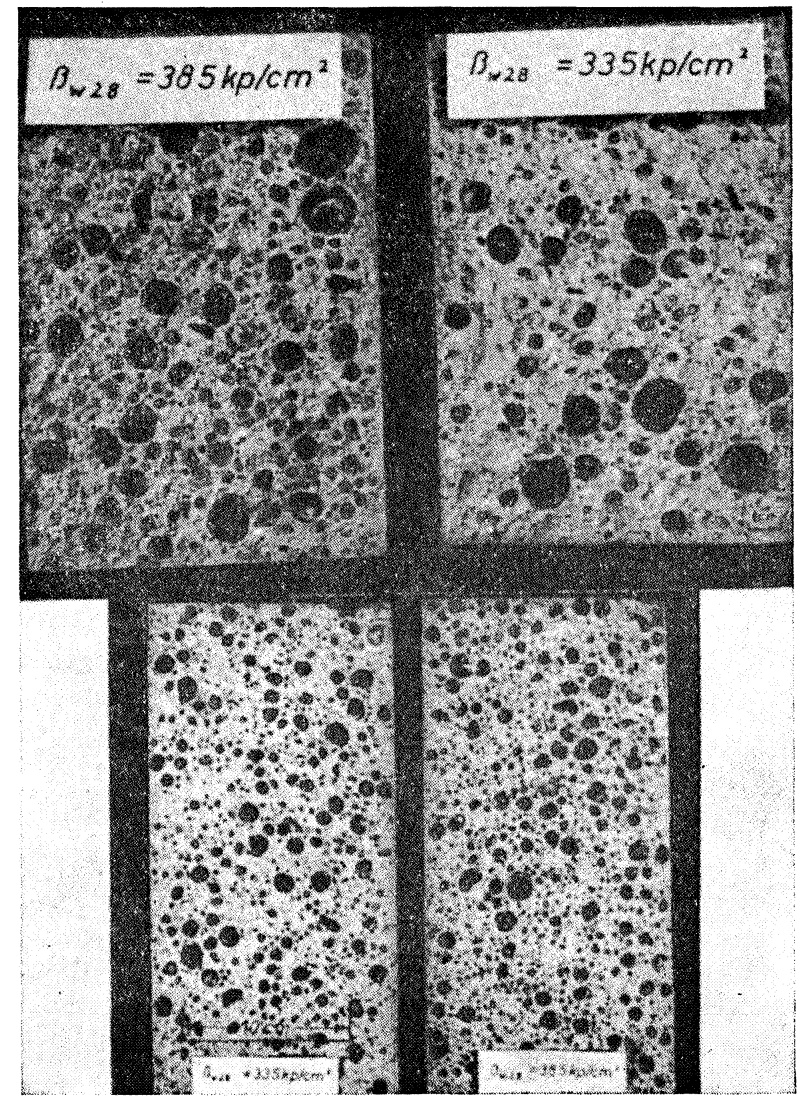

Fig. 3.-Planos de rotura del hormigón de arcillas dilatadas (Liapor $\eta$ ) con arena natural en una proporción de $30 \%$ del volumen de los áridos:

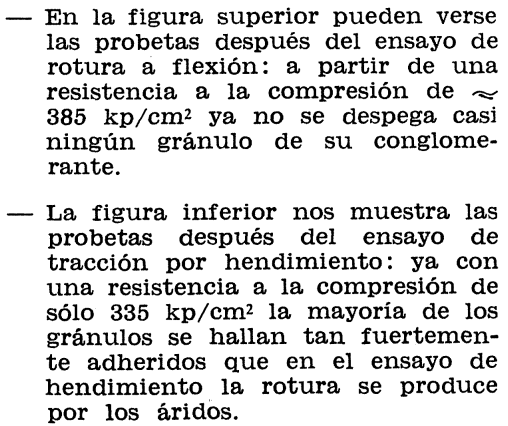

La figura 4 muestra la relación entre la resistencia a la compresión del hormigón $\beta_{\mathrm{w}}{ }^{20}$ y la resistencia a la tracción por flexión $\beta_{\mathrm{bz}}$ de probetas de $10 \times 15 \times 70 \mathrm{~cm}$ conservadas permanentemente húmedas en hormigones ligeros preparados con esquisto y arcilla dilatados como árido. Las curvas superior e inferior representan líneas límite de hormigo- 
nes normales con áridos triturados o áridos redondeados, según Hummel (5). La curva de rayas y puntos corresponde a la relación:

$$
\beta_{\mathrm{bz}}=\sqrt[3]{\beta_{\mathrm{w}}^{2}}
$$

que, según Hummel, puede servir como curva del valor medio de hormigón normal. En toda la zona examinada $\left(200 \mathrm{kp} / \mathrm{cm}^{2}<\beta_{\mathrm{w}}<600 \mathrm{kp} / \mathrm{cm}^{2}\right)$, las resistencias a la tracción por flexión de los hormigones ligeros se hallan preferentemente entre la curva del valor medio y la línea límite superior para hormigón normal. Sólo a partir de una calidad de hormigón de $500 \mathrm{kp} / \mathrm{cm}^{2}$ se encuentran, en forma creciente, algunos valores más desfavorables, pero que todavía se hallan muy por encima de la línea límite inferior. Por término medio, la resistencia a la tracción por flexión alcanzó aproximadamente los siguientes porcentajes de la resistencia a la compresión:

\begin{tabular}{|c|c|c|c|}
\hline $\begin{array}{c}\text { Resistencia } \\
\text { nominal }\end{array}$ & $\beta_{\mathrm{wm}}\left(\mathbf{k p} / \mathbf{c m}^{2}\right)$ & $\beta_{\mathrm{bz}}\left(\mathbf{k p} / \mathbf{c m}^{2}\right)$ & $\beta_{b z} / \beta_{w}(\%)$ \\
\hline LBn 150 & 200 & 38 & 19 \\
LBn 250 & 300 & .48 & 16 \\
LBn 350 & 400 & 58 & 14 \\
LBn 450 & 500 & 67 & 13 \\
LBn 550 & 600 & 73 & 12 \\
\hline
\end{tabular}

En total, la resistencia a la flexotracción aumenta más lentamente que la resistencia a la compresión. Esta tendencia crece al aumentar la calidad del hormigón.

Las resistencias a la tracción por flexión, incluidas en la figura 4, se obtuvieron después de un almacenamiento en húmedo. Si el hormigón se seca, a las tensiones aplicadas se superpone en la carga máxima un estado de tensión propia como consecuencia de la diferente retracción en las distintas zonas de la sección transversal. Las tensiones de retracción en las fibras marginales en el hormigón ligero pueden conducir, en gran medida, a una rotura prematura de los prismas de flexotracción si éstos no se encuentran perfectamente secos durante la prueba de resistencia. Debido al agua contenida en los poros de los gránulos de los áridos, el gradiente de humedad entre el núcleo y las zonas marginales en el hormigón ligero es a menudo mayor que en hormigón normal. Por esta causa, la resistencia a la flexotracción de hormigones ligeros en fase de secado disminuye la mayoría de las veces de modo desproporcionado frente a la de las probetas conservadas en húmedo. Sólo después de alcanzarse la humedad de compensación, vuelve otra vez a reproducirse la primitiva resistencia a la flexotracción. Esta compensación de humedad dura, no obstante, mucho tiempo sobre todo en hormigones ligeros con áridos humedecidos previamente.

La figura 5 presenta las resistencias a la tracción por hendimiento de hormigones ligeros con áridos de esquisto dilatado y arcilla expandida, según ensayos alemanes e ingleses.

El gráfico contiene además la zona de dispersión para hormigones ligeros, con y sin adición de arena natural, indicada por el Comité ACI 213 en (3) y las líneas-límite para la 
relación resistencia al hendimiento-resistencia a la compresión en cubos para hormigón normal según una recopilación de Bonzel (6). Se ve que la resistencia a la tracción por hendimiento correspondiente a una determinada resistencia a la compresión depende, en gran medida, de los áridos. Las resistencias al hendimiento logradas con el árido altamente resistente de esquisto dilatado "Norlit" se encuentran preferentemente dentro de la zona de dispersión del hormigón normal. Las parejas de valores obtenidas en hormigón de arcilla expandida "Aglite" y "Liapor", sin embargo, se agrupan alrededor del límite inferior, en tanto que las resistencias a la tracción por hendimiento del hormigón de esquisto dilatado Berwilit se hallan por debajo la mayoría de las veces, e incluso, a menudo, no alcanzan la línea-límite inferior de los hormigones ligeros americanos sin adición de arena natural.

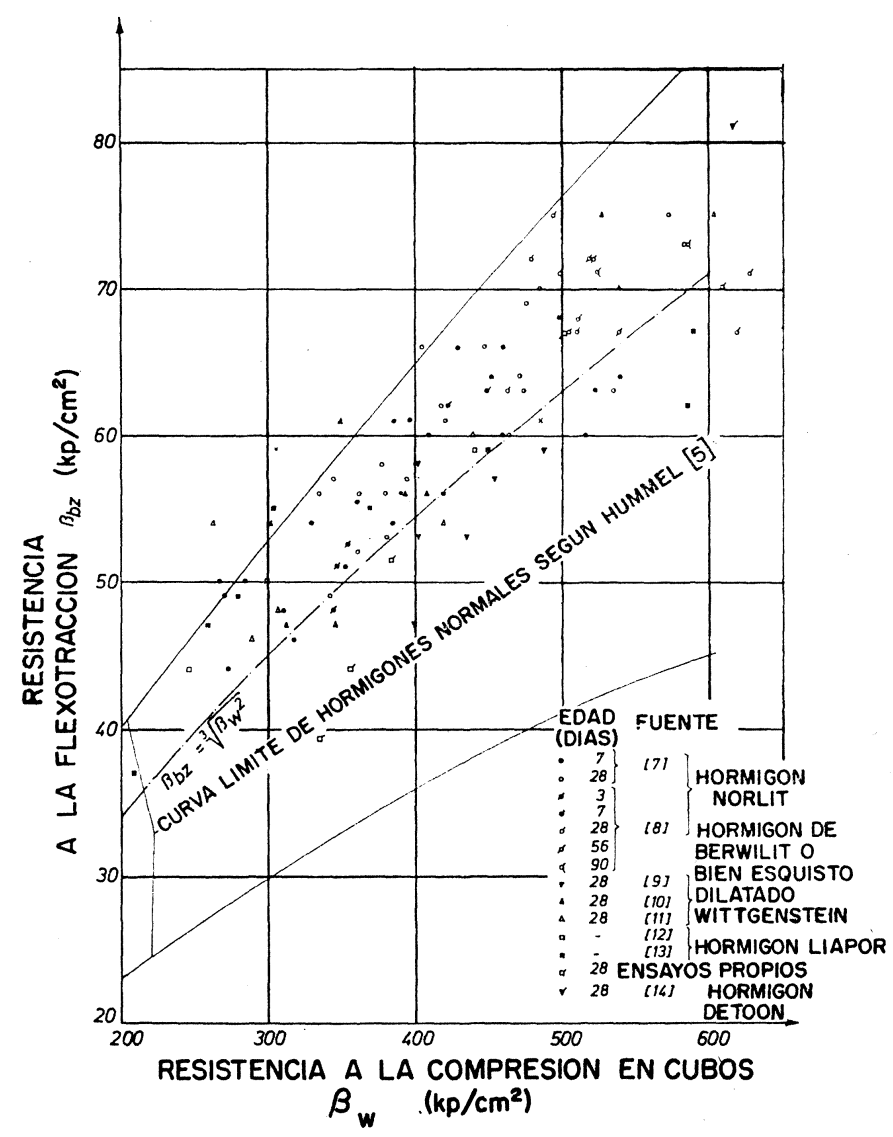

Fig. 4.-Relación entre resistencia a la compresión $\beta_{w}$ y la resistencia a la flexotracción $\beta_{b z}$ de hormigones ligeros cuyos áridos se componen de esquisto dilatado $y$ arcilla expandida.

En la figura 6 puede verse la relación entre la resistencia a la tracción por hendimiento y la resistencia a la flexotracción de hormigones de esquisto dilatado con áridos de resistencia media (Berwilit) y de gran resistencia (Norlit). En comparación con el hormigón normal [zona de dispersión según Bonzel (6)], para igual resistencia a la flexotracción en el hormigón ligero con Berwilit se encuentra una menor resistencia a la tracción por hendimiento. Los valores medidos en hormigones de Norlit corresponden aproximadamente a las relaciones en el hormigón normal. 


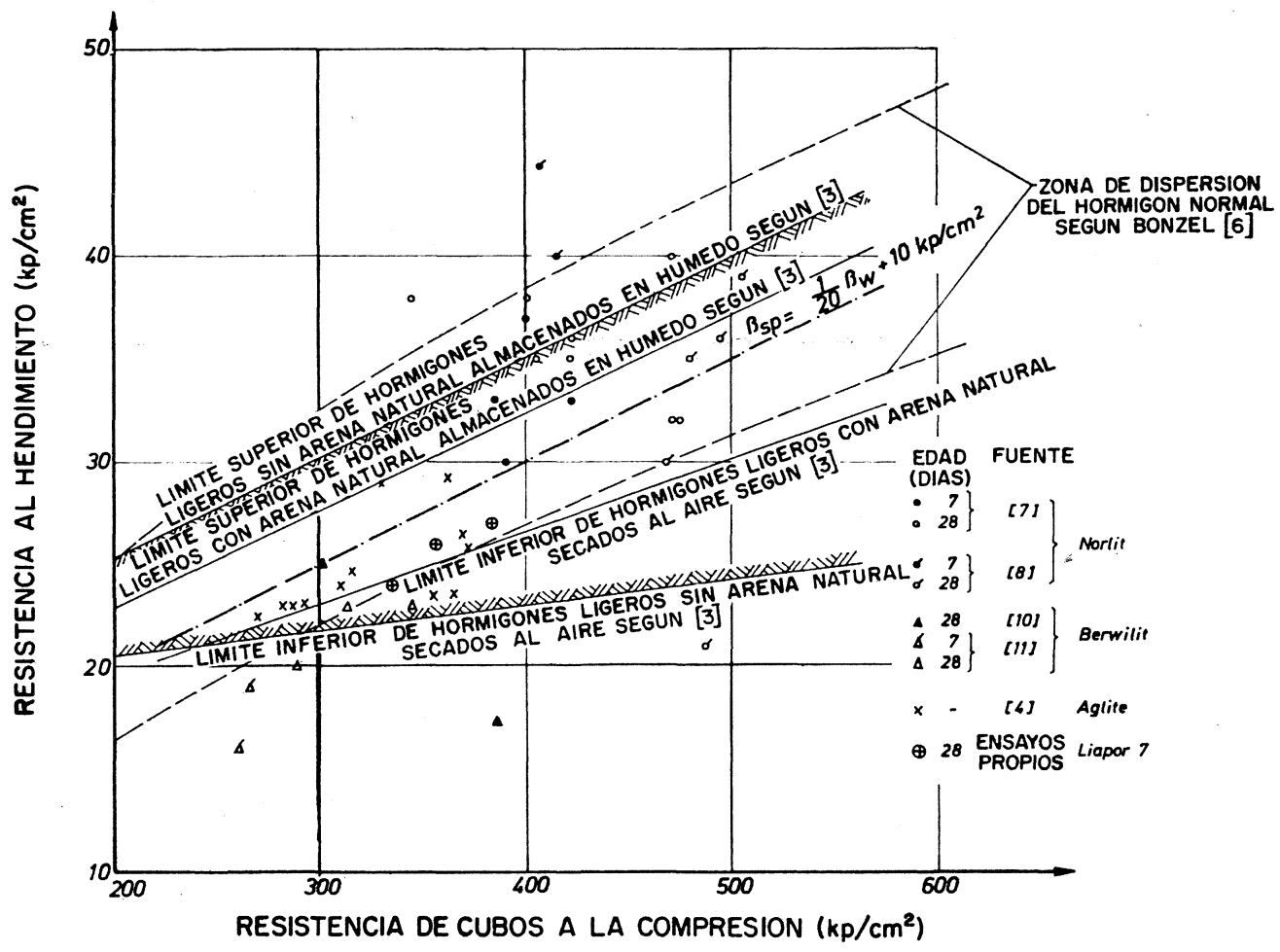

Fig. 5.-Relación entre la resistencia a la compresión en cubos y la resistencia a la tracción por hendimiento de hormigones ligeros.

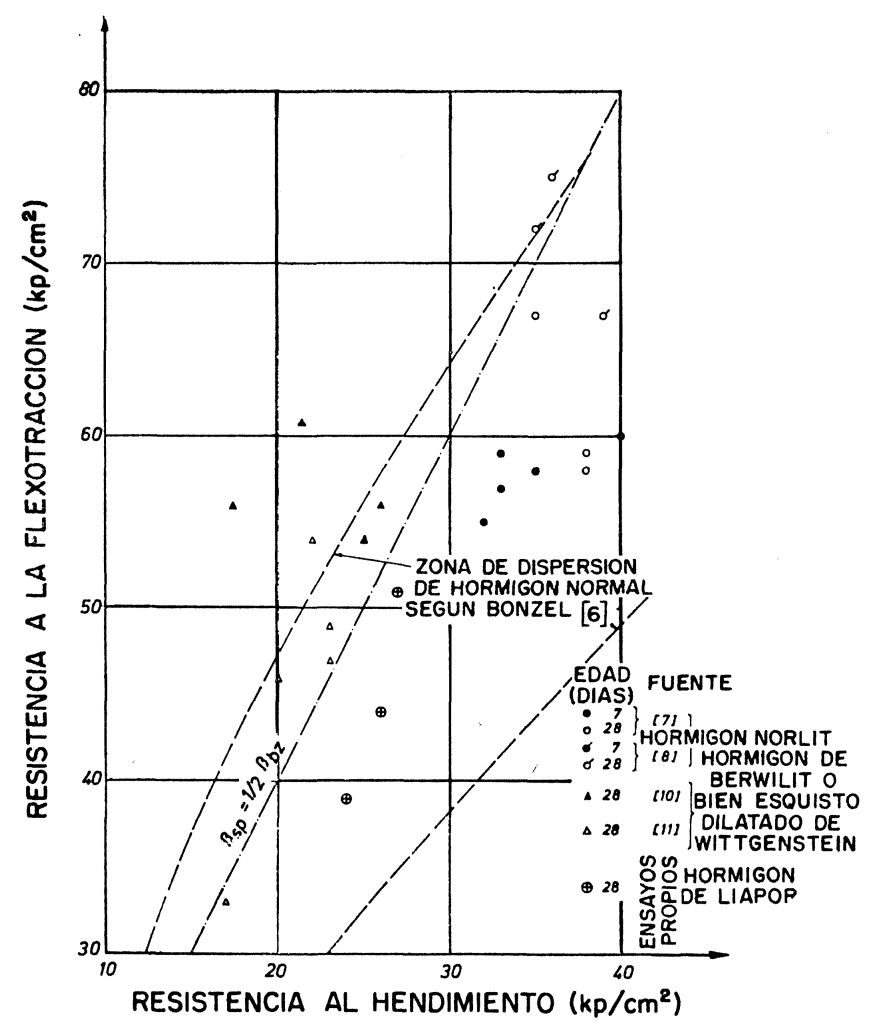

Fig. 6.-Relación entre la resistencia al hendimiento y la resistencia a flexotracción de hormigones de esquistos dilatados con áridos de resistencia media y gran resistencia. 
Eventuales tensiones previas como consecuencia de un secado irregular perturban menos la resistencia al hendimiento que la resistencia a la flexotracción. Las tensiones previas que se producen en el núcleo y en las zonas marginales debido a la retracción, son de sentido contrario al esfuerzo de la carga para hendimiento.

\section{BIBLIOGRAFIA}

(1) Walz, K., und G. WIschers: Konstruktionsleichtbeton hoher Festigkeit. Stand der Entwicklung in den USA am Beispiel von Blähtonzuschlag aus dem Drehofen. Beton 14 (1964) Heft 7, S. 293-298, Heft 8, S. 327-333 und Heft 9, S. 375-383.

(2) Wesche, K.: Stoffliche Grundlagen zum Entwurf von Leichtbetonkonstruktionen. Beton- und Stahlbetonbau 62 (1967) Heft 11, SS. 256-260.

(3) ACI Commitee 213: Guide for Structural Lightweight Aggregate Concrete. Journal of the American Concrete Institute, Proceedings 64 (1967), Heft 6, S. 433-469. Deutsche Bearbeitung: Betonsteinzeitung 33 (1967) Heft 12, Seite 569-581.

(4) SHort, A., and W. KINNIBURGH: Lightweight concrete, 2nd edition revised London: CR Books Ltd. 1968.

(5) Huminel, A.: Das Beton-ABC. Zwölfte Auflage. Verlag Wilhelm Ernst \& Sohn, Berlín, 1959.

(6) Bonzel, J.: Über die Spalzugfestigkeit del Betons. beton 14 (1964) Heft 3, S. 108-114 und Heft 4, S. 150-157.

(7) Nach einem amtlichen Prüfbericht des Instituts für Materialprüfung und Forschung des Bauwesens der Technischen Hochschule Hannover.

(8) Diemel, E., und H. Laporose: Erste Bundesstraßenbrücke in Leichtspannbeton LB 450. Beton- und Stahlbetonbau 63 (1968) Heft 7, S. 145-156.

(9) Mol, W. E., en A. J. M. Koemans: Vleugeldakplaten van voorgespannen licht beton. Cement XXI (1969) Heft 3, 112-120.

(10) Persönliche Mitteilung von Prof. Dr.-Ing. H. Beck.

(11) HeUfers, H., und H. AURICH: Beitrag zur Entwicklung des konstruktiven Leichtbetons in Deutschland. Technisch-wissensehaftliche Mitteilungen der Dyckerhoff-Zementwerke, Heft 3, S. 16.

(12) Liapor-Informationen der Fa. Lias-Leichtbaustoffe GmbH u. Co. KG, Schömberg.

(13) Nach amtlichen Prüfungsberichten des Otto-Graf-Instituts, Stuttgart.

(14) LAHL, W.: Beitrag zum Problem des Konstruktionsleichtbetons. Tonindustriezeitung 91 (1967) Nr. 9, S. $355-362$. 